\title{
A Nonlinear Model Predictive Concept for Control of Two-Phase Flows Governed by the Cahn-Hilliard Navier-Stokes System
}

\author{
Michael Hinze and Christian Kahle \\ Fachbereich Mathematik, \\ Optimierung und Approximation \\ Universität Hamburg, Bundesstraße 55, 20146 Hamburg, Germany \\ \{Michael.Hinze, Christian.Kahle\}@uni-hamburg.de
}

\begin{abstract}
We present a nonlinear model predictive framework for closed-loop control of two-phase flows governed by the Cahn-Hilliard Navier-Stokes system. We adapt the concept for instantaneous control from 61216 to construct distributed closed-loop control strategies for two-phase flows. It is well known that distributed instantaneous control is able to stabilize the Burger's equation [16] and also the Navier-Stokes system 612. In the present work we provide numerical investigations which indicate that distributed instantaneous control also is well suited to stabilize the Cahn-Hilliard Navier-Stokes system.
\end{abstract}

Keywords: Flow control, Navier-Stokes, Cahn-Hilliard, Model Predictive Control, Instantaneous Control, Adaptive Finite Elements.

\section{Introduction}

The aim of this work is the development of numerical methods for closed-loop control of two-phase flows governed by the Cahn-Hilliard Navier-Stokes system. The approach is based on an inexact variant of model predictive control called instantaneous control. Instantaneous control in the context of flow control is proposed in e.g. 5614, and for distributed control of the Navier-Stokes system is analyzed in 12, where among other things it is shown that the method is able to exponentially stabilize given solution states supposing certain smallness assumptions on the initial conditions. For an overview in the field of nonlinear model predicitve control we refer to [2021] and also to the monograph 9], where also further references can be found.

The outline of this paper is as follows. In section 2 we describe the concept of nonlinear model predictive control as it is used in the present work, and also introduce instantaneous control. In section 3 we present a brief introduction to the Cahn-Hilliard Navier-Stokes system, including its numerical treatment. In section 4 we discribe the instantaneous control strategy for the Cahn-Hilliard Navier-Stokes system and demonstrate its performance at morphing a circle into a square in section 5 . We end with some conclusions formulated in section 6 ]

D. Hömberg and F. Tröltzsch (Eds.): CSMO 2011, IFIP AICT 391, pp. 348 357, 2013.

(C) IFIP International Federation for Information Processing 2013 


\section{Nonlinear Model Predictive Control}

The aim of model predictive control consist in steering or keeping the state of a dynamical system to or at a given desired trajectory. To fix the concept, we are going to apply it to the Cahn-Hilliard Navier-Stokes system. Let us first consider an abstract dynamical system with initial condition $x_{0}$, state $x(t)$, observation $y(t)$ and control $u(t)$ :

$$
\begin{aligned}
\dot{x}(t)+A x(t) & =b(x, t)+\mathcal{B} u(t),(t>0) & & \text { state, } \\
y(t) & =\mathcal{C} x(t) & & \text { observation, } \\
x(0) & =x_{0} & & \text { initial condition. }
\end{aligned}
$$

Our aim consists in constructing a nonlinear feedback control law $K$ with $\mathcal{B} u(t)=$ $K(x(t))$ which steers the dynamical system to the desired trajectory $\bar{x}(t)$, i.e. $x(t) \stackrel{!}{\rightarrow} \bar{x}(t)(t \rightarrow \infty)$. To simplify notations we from here onwards use $\mathcal{B}=i d$ and $\mathcal{C}=i d$, i.e. we do not distinguish between state and observation and we allow fully distributed controls.

To prepare for model predicitve control, system (11) is discretized in time using the semi-implicit Euler method on an equidistant time grid $0=t_{0} \leq t_{1} \leq \ldots$ with $t_{k+1}-t_{k}=\tau$ for $k=0,1, \ldots$. Here $x^{k}$ denotes the state at time $t_{k}$ and $b^{k}$ denotes the nonlinearity $b\left(x^{k}, t_{k}\right)$. We obtain the time discrete model

$$
(I+\tau A) x^{k+1}=x^{k}+\tau b^{k}+u^{k+1}, \quad k=0,1, \ldots
$$

For $L \in \mathbb{N}$ and $x^{j}$ given, we consider the optimal control problem

$$
\begin{array}{r}
\min J\left(x^{j+1}, \ldots, x^{j+L}, u^{j+1}, \ldots, u^{j+L}\right) \\
\text { s.t. (2) for } j=k, \ldots, k+L-1,
\end{array}
$$

where

$$
J\left(x^{j+1}, \ldots, x^{j+L}, u^{j+1}, \ldots, u^{j+L}\right):=\sum_{i=1}^{L}\left(\frac{1}{2}\left\|x^{k+i}-\bar{x}^{k+i}\right\|^{2}+\frac{\alpha}{2}\left\|u^{k+i}\right\|^{2}\right)
$$

Let us note that for $L=1$ problem $\left(\overline{\mathcal{P}_{k}}\right)$ admits a unique solution. However, in the case $L>1$ the solution might not be unique due to the nonlinear character of the transition constraints (2). In this case we assume that $\left(\mathcal{P}_{k}\right.$ admits a solution.

Now we define the abstract model predictive control strategy using a computing oracle called RECIPE.

\section{Model Predicitve Control:}

1. Initialization: Specify time grid $\left(t_{j}\right)$ and discrete state $\bar{x}$, set $k=0$ and specify $L_{0}>0$.

2. Given $u^{k}, x^{k}$, set $u^{k+1}=R E C I P E\left(u^{k}, x^{k}, L_{k}\right)$. 
3. Solve (2) with $u^{k+1}$, i.e. compute $x^{k+1}$ according to $(I+\tau A) x^{k+1}=x^{k}+\tau b\left(x^{k}, t_{k}\right)+u^{k+1}$.

4. Set $k=k+1$, goto 2 ,

In the classical model predictive control context [2021] the oracle $R E C I P E(u, x, L)$, for given $u, x, L$, solves the optimal control problem $\left(\mathcal{P}_{k}\right)$ with $x^{k} \equiv x, u^{k} \equiv u$ and $L$ the length of the control horizon. From the solution $\left(x^{k+1}, \ldots, x^{k+L}, u^{k+1}, \ldots, u^{k+L}\right)$ only $x^{k+1}$ and $u^{k+1}$ are actually used to steer the discrete system to the next time instance $t_{k+1}$.

In practical applications often quick response to system changes is necessary. In such cases it may be too time consuming to solve problem $\left(\mathcal{P}_{k}\right)$ exactly. Instead, an approximate solution could be used. This leads to the so called concept of instantaneous control [6]12], whose oracle is described next.

\section{Instantaneous Control:}

Given $L, x, v$, then $u=\operatorname{RECIPE}(v, x, L)$ iff $u=u^{k+1}$, where $\left(x^{k+1}, \ldots, x^{k+L}, u^{k+1}, \ldots, u^{k+L}\right)$ is the result of a steepest descent step applied to the solution of $\left(\mathcal{P}_{k}\right)$ with $x^{k} \equiv x$ and $u^{k} \equiv v$.

In the case $L=1$, instantaneous control realizes the steps:

- Solve $(I+\tau A) z=x+\tau b\left(x, t_{k}\right)+v$,

- solve $\left(I+\tau A^{*}\right) \lambda=-(x-z)$,

- set $d=\alpha v-\lambda$,

- determine $\rho>0$ (step size for steepest descent),

- set RECIPE $=v-\rho d$,

where we have used the adjoint calculus to expose the derivations of the functional $J$, see e.g. [15].

Instantaneous control with $L=1$ is analytically investigated in [16] for the control of Burger's equation and in $[12$ for control of the two-dimensional NavierStokes system. Among other things it is shown in [12, Thm. 4.4,4.5] that

$$
\left\|x^{k}-\bar{x}^{k}\right\|_{H^{1}(\Omega)} \leq c \kappa^{k} \quad \text { for some } \kappa \in(0,1)
$$

and also that instantaneous control may be regarded as the discrete realization of a nonlinear feedback operator $K$ which is able to steer $x(t)$ exponentially fast to the desired trajectory $\bar{x}(t)$, i.e. with $u(t)=K(x(t))$ in (1) there holds

$$
\|x(t)-\bar{x}(t)\|_{H^{1}(\Omega)} \leq c \exp \left(-\frac{\rho}{\tau} t\right)
$$

where $\rho$ denotes the step size in the steepest descent algorithm and $\tau$ is the time step in the discretization, see [12, Thm. 4.1,4.2] for the details.

\section{The Cahn-Hilliard Navier-Stokes System}

The Cahn-Hilliard Navier-Stokes equations are a diffuse interface model for describing two-phase flows. In comparison to sharp interface models (see e.g. [8]) 
which model the interface between the two components as a sharp line, diffuse interface models allow a partial mixing of the components yielding a small diffuse interface. A derivation of the model used here can be found e.g. in [1]. It is related to the model ' $\mathrm{H}$ ' for two-phase flows in the classification of Hohenberg and Halperin [17]. We note that there are also models for flows with three components, see e.g [3].

For $\Omega \subset \mathbb{R}^{n},(n=2,3)$ and $T>0$ we here consider the following weak form of the Cahn-Hilliard Navier-Stokes system with double-obstacle free energy according to $[2$.

Find $(c(t), w(t), y(t), p(t))$ in $\mathcal{K} \times H^{1}(\Omega) \times H_{0}^{1}(\Omega) \times L_{(0)}^{2}(\Omega)$ such that

$$
\begin{array}{rlrl}
\left(\partial_{t} y, v\right)+\frac{1}{R e}(\nabla y: \nabla v) & & & \\
+((y \cdot \nabla) y, v)-(p, \operatorname{div} v)+(c \nabla w, v) & =0 & & \forall v \in H_{0}^{1}(\Omega), \text { a.e.t } \in(0, T] \\
(-\operatorname{div} y, v) & =0 & & \forall v \in L_{(0)}^{2}(\Omega), \text { a.e.t } \in(0, T], \\
c(t) & \in \mathcal{K} & & \text { a.e.t } \in(0, T], \\
\left(\partial_{t} c, v\right)+\frac{1}{P e}(\nabla w, \nabla v)-(c y, \nabla v) & =0 & \forall v \in H^{1}(\Omega), \text { a.e.t } \in(0, T] \\
\gamma^{2}(\nabla c, \nabla(v-c))-(w+c, v-c) & \geq 0 & \forall v \in \mathcal{K}, \text { a.e.t } \in(0, T] \\
c(x, 0) & =c^{0}(x) & \forall x \in \Omega \\
\partial_{\nu} c=0, \quad \partial_{\nu} w & =0 & & \text { on } \partial \Omega \times(0, T] \\
y(x, 0) & =y^{0}(x) & \forall x \in \Omega \\
y & =0 & & \text { on } \partial \Omega \times(0, T] .
\end{array}
$$

Here, for $v, w \in H^{1}(\Omega)$

$$
(\nabla v: \nabla w):=\int_{\Omega} \nabla v: \nabla w d x=\int_{\Omega} \sum_{i, j=1}^{n}(\nabla v)_{i j}(\nabla w)_{i j} d x
$$

and $c^{0} \in \mathcal{K}:=\left\{v \in H^{1}(\Omega)|| v \mid \leq 1\right.$ a.e. in $\left.\left.\Omega\right\}\right), y^{0} \in H_{0}^{1}(\Omega)$.

The function $c$ is called order parameter and satisfies $c=c(t, x) \in[-1,1]$, with $c \equiv 1$ on the pure $A$-phase and $c \equiv-1$ on the pure $B$-phase region, respectively, where $A$ and $B$ are the two components of the fluid. Initially, i.e. for $t=0$, we assume that the concentration equals $c^{0}$. The quantity $w$ represents the chemical potential, $y$ denotes the mean flow velocity field, i.e. $y=\frac{1+c}{2} y_{A}+\frac{1-c}{2} y_{B}$, where $y_{A}$ and $y_{B}$ denote the fluid velocities in the fluid phases $A$ and $B$, respectively, and $p \in L_{(0)}^{2}(\Omega)=\left\{v \in L^{2}(\Omega) \mid(v, 1)=0\right\}$ denotes the mean free pressure of the fluid. The flow profile at $t=0$ is given by $y^{0}$. The Péclet number $P e$ and the Reynolds number $R e$ are given physical constants. The parameter $\gamma$ is related to the width of the diffuse interface region which is of size $\mathcal{O}\left(\gamma^{2}\right)$ [4.

For an analytical treatment of the above system we refer to [1, Chapter 6.5]. Especially in two space dimensions there exists a unique solution $(c, w, y, p)$ to this system and we have 


$$
y \in C^{0}\left([0, T], \mathbf{H}_{0}^{1}(\Omega)\right), \quad c \in B C_{\omega}\left([0, T], H^{1}(\Omega)\right), \quad \nabla w \in L^{2}\left(0, T ; L^{2}(\Omega)\right) .
$$

Here, $B C_{\omega}\left([0, T], H^{1}(\Omega)\right)$ is the space of bounded and weakly continuous functions from $[0, T]$ with values in $H^{1}(\Omega)$.

\subsection{Time Discretization and Treatment of the Variational Inequality in (7)}

For the discretization of (3)-(11) we use the semi-implicit approach proposed in e.g. [7]19] with constant step size $\tau>0$. The variational inequality in (7) according to 10 is relaxed using Moreau-Yosida regularization. At time instance $t$ this results in finding $(y, p, c, w) \in H^{1}(\Omega)^{n} \times L_{(0)}^{2}(\Omega) \times H^{1}(\Omega) \times H^{1}(\Omega)$ such that

$$
\begin{aligned}
\left(y-y_{\text {old }}, v\right)+\frac{\tau}{R e}(\nabla y: \nabla v)+\tau\left(\left(y_{\text {old }} \cdot \nabla\right) y_{\text {old }}, v\right) & & & \\
+\tau\left(c_{\text {old }} \nabla w_{\text {old }}, v\right)-\tau(p, \operatorname{div} v) & =0 & & \forall v \in H_{0}^{1}(\Omega), \\
(-\operatorname{div} y, v) & =0 & & \forall v \in L_{(0)}^{2}(\Omega), \\
(c, v)+\frac{\tau}{P e}(\nabla w, \nabla v)-\tau\left(c_{\text {old }} y, \nabla v\right)-\left(c_{\text {old }}, v\right) & =0 & & \forall v \in H^{1}(\Omega), \\
\gamma^{2}(\nabla c, \nabla v)-(w, v)+\left(\lambda_{s}(c), v\right)-\left(c_{\text {old }}, v\right) & =0 & & \forall v \in H^{1}(\Omega) .
\end{aligned}
$$

is satisfied. Here the subscript old refers to the value of the respective function at time $t_{\text {old }}=t-\tau$ and $\lambda_{s}(c)=\lambda_{s}^{+}(c)+\lambda_{s}^{-}(c)=s(\max (0, c-1)+\min (0, c+1))$ stems from Moreau-Yosida regularization of (7), see e.g. [10/11] for details. Let us emphasize that (12)-(15) is decoupled in the sense that using $y_{\text {old }}, c_{\text {old }}$ and $w_{\text {old }}$, the flow $y$ and the pressure $p$ at time $t$ can be computed from (12)-(13), and then using this flow vector $y$, the concentration $c$ and the chemical potential $w$ are obtained from (14)-(15). Furthermore, normalizing $p$ by $(p, 1)=0$, it can be shown that (12)-(15) admits a unique solution $(y, p, c, w)$, compare [11, Thm 4.1]. The system (14)-(15) is nonlinear and can be treated by semi-smooth Newton methods, see [11].

\subsection{Spatial Discretization}

The spatial discretization is performed by linear finite elements for both the concentration and the chemical potential to obtain finite element approximations $c^{h}, w^{h}$. For the flowfield and the pressure we use the LBB-stable Taylor-Hood $P^{2}-P^{1}$ finite elements, see e.g. [18|22], to obtain finite element approximations $y^{h}, p^{h}$. For the spatial treatment of the Cahn-Hilliard part (14)-(15) we use the adaptive approach presented in 10:11. We emphasize that we use different spatial meshes for the Cahn-Hilliard and the Navier-Stokes part. 


\section{Instantaneous Control of the Cahn-Hilliard Navier-Stokes System}

The aim of this section is to develop a simple distributed closed-loop control strategy for the Cahn-Hilliard Navier-Stokes system. It uses instantaneous control with $L=1$ on a control horizon which coincides with the time interval of one time step. We consider the idealized situation that the flow can be controlled by a vector field which is distributed over the whole spatial domain and that the concentration $c$ can be measured in the whole spatial domain. The control goal consists in steering the concentration $c$ towards a prescribed concentration trajectory $c_{d}$ by applying volume forces to the flow field.

Now let $t_{0}=0$ and $k \in \mathbb{N}$. At time instance $t=t_{k}$, for $\alpha>0$, we consider the minimization problem

$$
\min J_{k}(u):=\frac{1}{2}\left\|c-c_{d}\right\|_{L^{2}(\Omega)}^{2}+\frac{\alpha}{2}\|u\|_{L^{2}(\Omega)}^{2}
$$

s.t.

$$
\begin{aligned}
\left(y-y_{\text {old }}, v\right)+\frac{\tau}{R e}(\nabla y: \nabla v)+\tau\left(\left(y_{\text {old }} \cdot \nabla\right) y_{\text {old }}, v\right) & & & \\
+\tau\left(c_{\text {old }} \nabla w_{\text {old }}, v\right)-\tau(p, \operatorname{div} v) & =u & & \forall v \in H_{0}^{1}(\Omega), \\
(-\operatorname{div} y, v) & =0 & & \forall v \in L_{(0)}^{2}(\Omega), \\
(c, v)+\frac{\tau}{P e}(\nabla w, \nabla v)-\tau\left(c_{\text {old }} y, \nabla v\right)-\left(c_{\text {old }}, v\right) & =0 & & \forall v \in H^{1}(\Omega), \\
\gamma^{2}(\nabla c, \nabla v)-(w, v)+\left(\lambda_{s}\left(c_{o l d}\right), v\right)-\left(c_{\text {old }}, v\right) & =0 & & \forall v \in H^{1}(\Omega) .
\end{aligned}
$$

where now old refers to the time instance $t_{k-1}$.

It is not hard to show that problem $\left(\mathcal{P}_{k}\right.$ admits a unique solution $u \in L^{2}(\Omega)^{n}$, which together with $(y, p, c, w)$ satisfies the adjoint system

$$
\begin{array}{rlrl}
\left(p_{1}, v\right)+\gamma^{2}\left(\nabla p_{2}, \nabla v\right) & =\left(c-c_{d}, v\right) & & \forall v \in H^{1}(\Omega), \\
\left(p_{2}, v\right) & =\frac{\tau}{P e}\left(\nabla p_{1}, \nabla v\right) & \forall v \in H^{1}(\Omega), \\
\left(p_{3}, v\right)+\frac{\tau}{R e}\left(\nabla p_{3}, \nabla v\right)-\tau\left(p_{4}, \operatorname{div} v\right) & =\tau\left(p_{1} \nabla c_{o l d}, v\right) & & \forall v \in\left(H_{0}^{1}(\Omega)\right)^{n}, \\
\left(\operatorname{div} p_{3}, v\right) & =0 & & \forall v \in L_{(0)}^{2}(\Omega), \\
\alpha u+p_{3} & =0 . & &
\end{array}
$$

i.e. there exists a uniquely determined adjoint $\left(p_{1}, p_{2}, p_{3}, p_{4}\right) \in H^{1}(\Omega) \times H^{1}(\Omega) \times$ $H^{1}(\Omega)^{n} \times L_{(0)}^{2}(\Omega)$ which solves (20)-(24). The gradient of $J_{k}$ is given by $\nabla J_{k}(v)=$ $\alpha v+p_{3}$. Its evaluation for a given $v \in L^{2}(\Omega)^{n}$ amounts to first solving (16) - (19) for $(y, p, c, w)$ and then solving (20)-(23) for $\left(p_{1}, p_{2}, p_{3}, p_{4}\right)$.

Let us note that (16)-(19) differ from (12)-(15) in the explicit treatment of the nonlinearity $\lambda_{s}$, which in (19) is frozen at $t_{\text {old }}$. We emphasize that (16)(19) is not used to simulate the controlled Cahn-Hilliard Navier-Stokes system, but to construct a feedback operator $K$ such that $u=K(y, p, c, w)$. With this 
feedback operator available, system (3)-(11) then is controlled through inserting $u=K(y, p, c, w))$ as right hand in (3). The resulting system then is treated on the time discrete level as in (12)-(15), where $K$ is evaluated at $t_{\text {old }}$, i.e.

$$
u=K\left(y_{o l d}, p_{\text {old }}, c_{\text {old }}, w_{\text {old }}\right) .
$$

Next we describe the construction of $K$ from (16)-(24). For this pupose let us denote by $B$ the solution operator associated to the quasi-Stokes problem (12)(13) and by $\mathcal{C}$ the linear, fourth order solution operator of the Cahn-Hilliard system (18)-(19). Then the system to obtain $p_{3}$ can be written in the form

$$
\begin{aligned}
y & \left.=B\left(y_{\text {old }}-\tau\left(y_{\text {old }} \nabla y_{\text {old }}\right)\right)-\tau c_{\text {old }} \nabla w_{\text {old }}+u\right), \\
c & =\mathcal{C}\left(c_{\text {old }}-\tau y \nabla c_{\text {old }}+\frac{\tau}{P e} \Delta\left(\lambda_{s}\left(c_{\text {old }}\right)-c_{\text {old }}\right)\right), \\
p_{1} & =\mathcal{C}\left(c-c_{d}\right), \text { and } \\
p_{3} & =-\tau B\left(p_{1} \nabla c_{\text {old }}\right) .
\end{aligned}
$$

Using these abbreviations, the control $u$ obtained by the instantaneous control strategy for $u_{0}^{k}=0$ is given by

$$
\begin{aligned}
\tilde{y} & =B\left(y_{\text {old }}+\tau b\left(y_{\text {old }}\right)-\tau c_{\text {old }} \nabla w_{\text {old }}\right), \\
\tilde{c} & =\mathcal{C}\left(c_{\text {old }}-\tau \nabla c_{\text {old }} \tilde{y}+\frac{\tau}{P e} \Delta\left(\lambda_{s}\left(c_{\text {old }}\right)-c_{\text {old }}\right)\right), \\
u & =\rho \tau B \nabla c_{\text {old }} \mathcal{C}\left(\tilde{c}-c_{d}\right)=: K\left(y_{\text {old }}, p_{\text {old }}, w_{\text {old }}, w_{\text {old }}\right)
\end{aligned}
$$

and is inserted in (12).

Note that this system does not depend on $\alpha$ since $u_{0}^{k}=0$.

The spatially discrete treatment of (12)-(15) with this control is similar as in the uncontrolled case. We note that (24) motivates to use Taylor-Hood finite elements for the discretization of the control, see the concept of variational discretization proposed in 13 .

\section{Numerical Results - Circle2Square}

To demonstrate the effectiveness of our control method we now morph a circle into a square with the following setup. As domain we use $\Omega:=(0,1)^{2}$, the initial concentration $c_{0}$ is chosen as 1 in $B_{\frac{1}{4}}\left(\frac{1}{2}, \frac{1}{2}\right)$ and as -1 in $\Omega \backslash B_{\frac{1}{4}}\left(\frac{1}{2}, \frac{1}{2}\right)$, see Fig. 1 (left). Control is applied to the flow field with control gain of steering $c$ to the desired state $c_{d}$ with values 1 in the square centered at $\left(\frac{7}{20}, \frac{7}{20}\right)$, see Fig. 1 (right), with edge length such that $\left(c_{0}, 1\right)=\left(c_{d}, 1\right)$. This requirement is meaningful, since our time discretization scheme is mass-conserving. We choose $R e=10, P e=100, \gamma=1 /(40 \pi), \alpha=1 e-4, \tau=0.01$ and use $\rho=1$ as step size in the steepest descent method.

Fig. 2 presents snapshots of the concentration after 40 time steps (left) and after 500 time steps (right, where also the controlled flow is depicted). Clearly, the corners formed in $c_{d}$ cannot be reached by the controlled concentration, since 

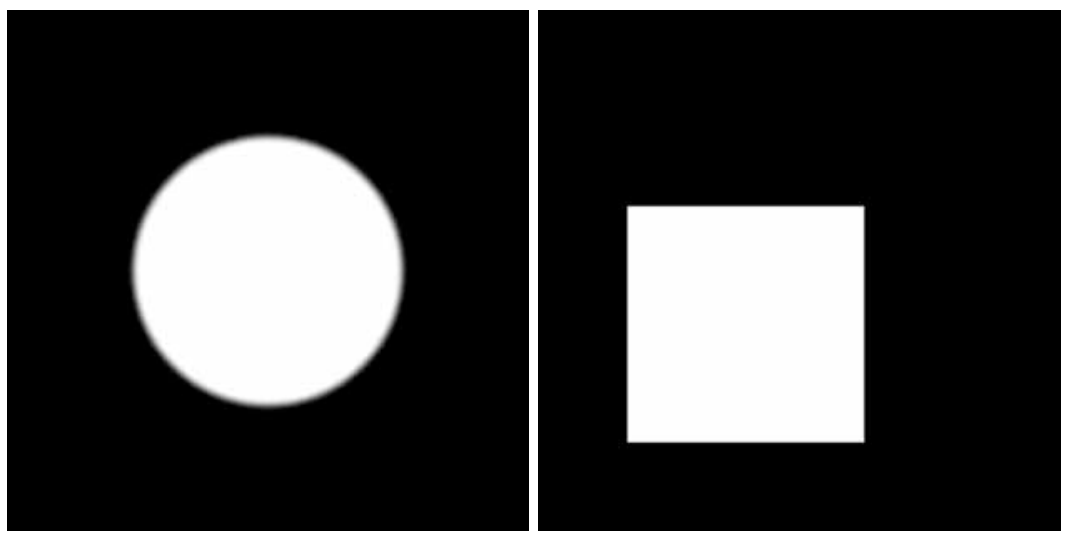

Fig. 1. Initial state $c_{0}$ (left) and desired state $c_{d}$ (right). Black indicates -1 , white indicates +1 .

the phasefield approach used here always delivers a smooth diffuse interface in the present situation. Fig. 3 presents the evaluation of $d(t):=\left\|c(t)-c_{d}(t)\right\|_{L^{2}(\Omega)}$ for various Reynolds numbers ranging in the interval [10,200]. We observe a clear decrease in $d(t)$, till a certain value around $1 e-1$ is reached. The method is not able to further reduce $d(t)$ due to the unreachability of $c_{d}$. The oscillations of $d$ for larger Reynolds numbers can be explained by the indirect control method (flow is controlled, concentration should be steerd to $c_{d}$ ), which becomes more sensible with increasing Reynolds number.
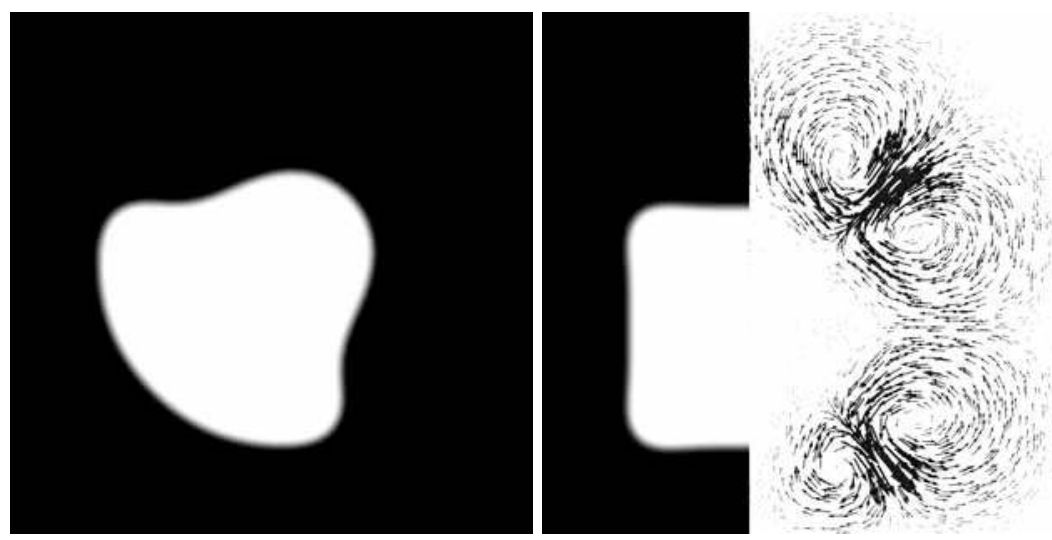

Fig. 2. Controlled state at $t=40 \tau$ and $t=500 \tau$ 


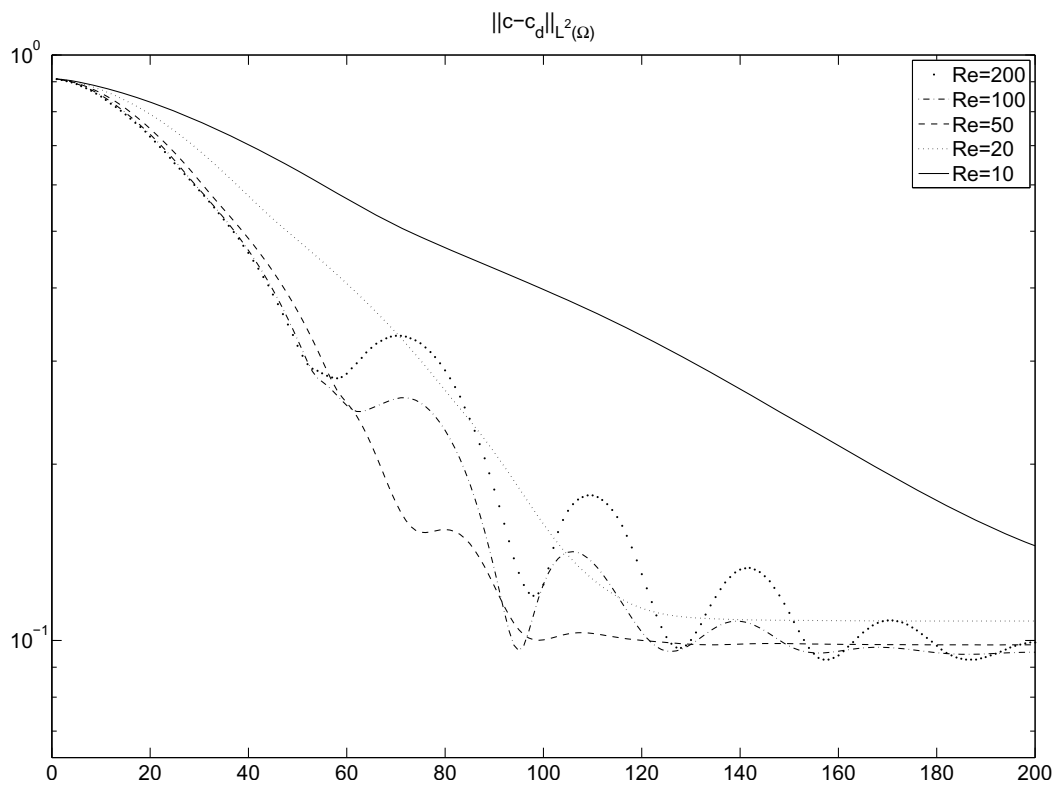

Fig. 3. The reduction of $\left\|c-c_{d}\right\|$ for various Reynolds numbers

\section{Conclusion}

We have presented a general inexact model predictive control concept called instantaneous control and sketched its interpretation as closed loop controller. For the Cahn-Hilliard Navier-Stokes system we have derived a nonlinear feedback $u=K(y, p, c, w)$ which realizes instantaneous control on the continuous level. With morphing the circle into a square we have numerically demonstrated the scope and effectiveness of our approach in control of two-phase flows.

\section{References}

1. Abels, H.: Diffuse interface models for two-phase flows of viscous incompressible fluids. Max-Planck Institut für Mathematik in den Naturwissenschaften, Leipzig, Lecture Note no.: 36 (2007)

2. Blowey, J.F., Elliott, C.M.: The Cahn-Hilliard gradient theory for phase separation with non-smooth free energy. Part I: Mathematical analysis. European Journal of Applied Mathematics 2, 233-280 (1991)

3. Boyer, F., Lapuerta, C., Minjeaud, S., Piar, B., Quintard, M.: CahnHilliard/Navier-Stokes model for the simulation of three-phase flows. Transp. Porous Media 82(3), 463-483 (2010)

4. Cahn, J.W., Hilliard, J.E.: Free energy of a nonuniform system-I: Interfacial free energy. J. Chem. Phys. 28, 258-267 (1958) 
5. Choi, H.: Suboptimal control of turbulent flow using control theory. In: Proceedings of the International Symposium on Mathematical Modelling of Turbulent Flows, Tokyo, Japan (1995)

6. Choi, H., Hinze, M., Kunisch, K.: Instantaneous control of backward-facing step flows. Applied Numerical Mathematics 31(2), 133-158 (1999)

7. Eyre, D.J.: Unconditionally gradient stable time marching the Cahn-Hilliard equation. In: Computational and Mathematical Models of Microstructural Evolution. MRS Proceedings, vol. 529 (1998)

8. Gross, S., Reusken, A.: Numerical methods for two-phase incompressible flows. Springer Series in Computational Mathematics, vol. 40. Springer (2011)

9. Grüne, L., Pannek, J.: Nonlinear Model Predictive Control. Communications and Control Engineering. Springer (2011)

10. Hintermüller, M., Hinze, M., Kahle, C.: An adaptive finite element Moreau-Yosidabased solver for a coupled Cahn-Hilliard/Navier-Stokes system (preprint, submitted for publication)

11. Hintermüller, M., Hinze, M., Tber, M.H.: An adaptive finite element MoreauYosida-based solver for a non-smooth Cahn-Hilliard problem. Optimization Methods and Software 25(4-5), 777-811 (2011)

12. Hinze, M.: Instantaneous closed loop control of the Navier-Stokes system. SIAM J. Contr. Optim. 44(2), 564-583 (2005)

13. Hinze, M.: A variational discretization concept in control constrained optimization: the linear quadratic case. Computational Optimization and Applications 30(1), 45-61 (2005)

14. Hinze, M., Kunisch, K.: Three control methods for time - dependent Fluid Flow. Flow, Turbulence and Combustion, vol. 60, pp. 273-298. Springer (2000)

15. Hinze, M., Pinnau, R., Ulbrich, M., Ulbrich, S.: Optimization with PDE constraints. Springer, Heidelberg (2009)

16. Hinze, M., Volkwein, S.: Instantaneous control for the Burgers equation: Convergence analysis and numerical implementation. Nonlinear Analysis 50(1), 1-26 (2002)

17. Hohenberg, P.C., Halperin, B.I.: Theory of dynamic critical phenomena. Rev. Mod. Phys. 49(3), 435-479 (1977)

18. Hood, P., Taylor, G.: Navier-Stokes equations using mixed interpolation. Finite Element Methods in Flow Problems. UAH Press (1974)

19. Kay, D., Styles, V., Welford, R.: Finite element approximation of a Cahn-HilliardNavier-Stokes system. Interfaces and Free Boundaries 10(1), 15-43 (2008)

20. Nevistic, V., Primbs, J.A.: Finite Receding Horizon Control: A General Framework for Stability and Performance Analysis. Technical Report 6, Automatic control laboratory, ETH Zürich (1997)

21. Qin, S.J., Badgwell, T.A.: A survey of industrial model predictive control technology. Control Engineering Practice 11, 733-764 (2003)

22. Verfürth, R.: A posteriori error analysis of space-time finite element discretizations of the time-dependent Stokes equations. Calcolo 47, 149-167 (2010) 